\title{
Successful Treatment of an Immune-Mediated Colitis Induced by Checkpoint Inhibitor Therapy in a Patient with Advanced Melanoma
}

\author{
Maria Paparoupa $^{a} \quad$ Sophie Stupperich $^{b} \quad$ Lisa Goerg-Reifenberg ${ }^{c}$ \\ Andreas Wittig ${ }^{\mathrm{b}}$ Frank Schuppert ${ }^{\mathrm{b}}$ \\ aDepartment of Intensive Care Medicine, University Medical Center Hamburg-Eppendorf, \\ Hamburg, Germany; ${ }^{b}$ Department of Gastroenterology, Endocrinology, Diabetology and \\ General Medicine, Klinikum Kassel, Kassel, Germany; 'Department of Dermatology, \\ Klinikum Kassel, Kassel, Germany
}

\section{Keywords}

Immune checkpoint inhibitor · Nivolumab - Ipilimumab · Infliximab · Immune-related adverse events

\begin{abstract}
Immune checkpoint inhibitors (ICls) have been used as immunotherapeutic agents in several malignancies because of their ability to modify the $T$ cell-mediated response against tumor cells. Dual checkpoint inhibition improves remission rates in patients with metastatic melanoma compared to monotherapy. However, a higher incidence of toxicity, including immunerelated colitis, has been reported before. A 54 -year-old female was diagnosed with malignant melanoma on her left upper arm. Because of progressive metastatic disease, a rescue therapy with nivolumab (Opdivo ${ }^{\circledR}$ ) $1 \mathrm{mg} / \mathrm{kg}$ and ipilimumab (Yervoy ${ }^{\circledR}$ ) $3 \mathrm{mg} / \mathrm{kg}$ was initiated and a clinical and radiological remission was achieved. Two weeks after completing the third cycle of the $\mathrm{ICl}$ therapy, the patient presented with persistent hemorrhagic diarrhea, nausea and abdominal pain. A diagnostic colonoscopy revealed multiple ulcerative lesions and hemorrhagic colitis of the sigmoid and rectum. Due to the ongoing treatment with nivolumab and ipilimumab, the diagnosis of a checkpoint inhibitor-induced colitis was made and
\end{abstract}




\section{Case Reports in Gastroenterology}

Case Rep Gastroenterol 2020;14:554-560 DOI: 10.1159/000511252

(c) 2020 The Author(s). Published by S. Karger AG, Basel www.karger.com/crg

Paparoupa et al.: Checkpoint Inhibitor-Induced Colitis

immunosuppression with local and systemic steroids, such as mesalazine was initiated. In order to achieve a long-lasting steroids reduction, we decided to start with infliximab (Remicade ${ }^{\circledR}$ $5 \mathrm{mg} / \mathrm{kg}$ body weight i.v. every 2 weeks). Clinical remission was achieved and prednisolone could be subsequently discontinued. Infliximab, in combination with mesalazine, could successfully induce a long-lasting remission without steroids. The treatment of $\mathrm{ICl}$-induced colitis did not lead to a reoccurrence of malignant melanoma after 2 years of follow-up.

(C) 2020 The Author(s)

Published by S. Karger AG, Basel

\section{Introduction}

Immune checkpoint inhibitors (ICIs) are becoming increasingly important as therapeutic agents in several malignancies because of their ability to modify the T cell-mediated immune response against tumor cells. The programmed death-1 co-receptor (PD-1) or ligand (PD-L1) and the cytotoxic $\mathrm{T}$ lymphocyte-associated protein 4 (CTLA-4) are well-established checkpoint targets. Nivolumab, an IgG4 anti-PD-1 antibody, and ipilimumab, an anti-CTLA-4 antibody, have been approved as a rescue therapy in advanced melanoma, leading to rapid and long-term durable clinical responses [1].

A number of immune-related adverse events (irAEs) are associated with the use of ICIs. Clinicians using these agents should be aware of the spectrum and the incidence of irAEs, in order to recognize and treat them properly. According to a study by Almutairi et al. [2] in patients with advanced melanoma receiving ICIs, ipilimumab was related to irAEs affecting the gastrointestinal system (diarrhea, 29\%; and colitis, 8\%), skin (rash, 31\%; pruritus, 27\%; and dermatitis, $10 \%$ ) and the hypophysis (hypophysitis, $4 \%$ ), while nivolumab caused more often maculopapular rash (13\%), erythema (4\%), hepatitis (3\%), and infusion-related reactions (3\%) [2]. Our group has recently published a study describing the wide clinical spectrum of autoimmune hypophysitis related to the use of checkpoint inhibitors [3].

Immune-related adverse events affecting the digestive system are colitis, hepatitis and pancreatitis. A T cell-mediated immune response is probably involved in the ICI-induced colitis, as infiltration with CD8 ${ }^{+}$cells and T-bet expressing $\mathrm{CD} 4^{+}$cells has been observed in the colon biopsies of patients receiving nivolumab [4]. A meta-analysis by Tian et al. [5] reported an increased incidence risk of all grade colitis in patients with solid tumors receiving ICIs, compared to those undergoing chemotherapy or chemotherapy and ICIs. The lower incidence risk was observed under monotherapy with nivolumab [5].

Steroids are generally recommended as first-line treatment against all kinds of ICI-induced adverse events [6]. Infliximab and vedolizumab have been successfully used in patients with immune-mediated colitis, being refractory to steroids [7, 8]. However, data regarding the side effects of the concomitant administration of nivolumab and ipilimumab are rare and prospective, controlled clinical trials are still lacking in this field. Furthermore, it is generally believed that immunosuppression may adversely affect the antitumor efficacy of the ICIs therapy [9].

We describe the case of a patient with advanced melanoma treated with nivolumab and ipilimumab and who subsequently developed a steroid-refractory immune-mediated colitis. The patient was successfully treated with infliximab and mesalazine and has remained progression-free for 2 years. 


\section{Case Reports in Gastroenterology}

Case Rep Gastroenterol 2020;14:554-560

DOI: 10.1159/000511252

c) 2020 The Author(s). Published by S. Karger AG, Basel www.karger.com/crg

Paparoupa et al.: Checkpoint Inhibitor-Induced Colitis

\section{Case Presentation}

A 54-year-old otherwise healthy female was diagnosed with a malignant melanoma on her left upper arm. The lesion was entirely removed with a margin of $2 \mathrm{~cm}$. After 6 months on adjuvant therapy with interferon alpha-2a $\left(\right.$ Roferon $\left.{ }^{\circledR}\right)$, progressive metastatic disease was observed. A radical axillary lymphadenectomy and radiation of the left axillary region (50 Gray) were performed. The patient then developed a mild autoimmune hyperthyroidism with subsequent hypothyroidism, requiring substitution therapy with L-thyroxine $75 \mu \mathrm{g} / \mathrm{day}$. An autoimmune insufficiency of the pituitary gland was excluded by testing all five regulatory pathways. During restaging, she presented with disseminated lung, liver and peritoneal metastasis, forming the indication for a rescue therapy with nivolumab (Opdivo ${ }^{\circledR}$ ) $1 \mathrm{mg} / \mathrm{kg}$ and ipilimumab (Yervoy $\left.{ }^{\circledR}\right) 3 \mathrm{mg} / \mathrm{kg}$ and discontinuation of interferon alpha-2a.

After three cycles of nivolumab and ipilimumab in the course of 3 months, restaging investigations still showed pulmonary, hepatic and lymphatic metastases. In addition, the patient presented with persistent hemorrhagic diarrhea, nausea and abdominal pain 2 weeks after completing the third cycle of ICI therapy. A diagnostic colonoscopy revealed multiple ulcerative lesions and hemorrhagic colitis of the sigmoid and rectum (Fig. 1). The rest of the colon showed edematous mucosa, while the terminal ileum and ileocecal valve were macroscopically intact (Fig. 2). A series of sequential mucosal biopsies were taken from the proximal to the distal colon. Histological analysis confirmed an acute and chronic inflammatory mucosal alteration which was compatible with inflammatory bowel disease (IBD) with a high apoptosis rate. Calprotectin was elevated to $>800 \mu \mathrm{g} / \mathrm{g}$ stool. Due to the ongoing treatment with nivolumab and ipilimumab, the diagnosis of an ICI-induced colitis was made.

After excluding an infection with cytomegalovirus by RT-PCR in serum and colonic tissue, an immunosuppression with local hydrocortisone acetate (Colifoam ${ }^{\circledR}$ rectal foam once daily) and oral steroids (prednisolone $80 \mathrm{mg}$ daily in the morning) was initiated. Furthermore, the patient received mesalazine locally (Salofalk ${ }^{\circledR}$ Enema $4 \mathrm{~g} / 60 \mathrm{~mL}$ once daily in the evening) and orally (Salofalk ${ }^{\circledR}$ gastro-resistant prolonged-release granules $3 \mathrm{~g}$ once daily in the morning).

After a short clinical remission of 2 months, symptoms relapsed when a reduction of systemic steroids below $30 \mathrm{mg}$ daily was attempted. Prednisolone was increased to $150 \mathrm{mg} /$ day and symptoms improved. It was slowly tapered again, but symptoms related to a flare-up of the colonic inflammation, like bloody diarrhea, nausea and abdominal pain, relapsed after reaching a dose of less than $30 \mathrm{mg} /$ day. Positively, 6 months after discontinuation of nivolumab and ipilimumab, radiological remission of metastatic disease was documented for the first time.

In order to induce remission of the colitis without long-term steroid therapy, we decided to start an immunosuppressive therapy with infliximab $\left(300 \mathrm{mg}\right.$ Remicade $^{\circledR}, 5 \mathrm{mg} / \mathrm{kg}$ body weight i.v. every 2 weeks). Infliximab was combined with the previously started corticosteroid and mesalazine therapy. Prednisolone was gradually reduced to $30 \mathrm{mg} /$ day. Already $1 \mathrm{month}$ after initiation of infliximab, our patient was free of symptoms. Two weekly cycles of intravenous infliximab were continued for 6 months until the next routine examination, where calprotectin was in the normal range ( 6 months earlier $>800 \mu \mathrm{g} / \mathrm{g}$ stool) and remission of metastatic disease was still in place. At the same time, prednisolone could be gradually reduced to a minimum of $2.5 \mathrm{mg} /$ day and subsequently discontinued altogether. During endoscopic follow-up, only minimal residual inflammation was seen, so that a 4-weekly scheme of infliximab was decided. 


\section{Case Reports in Gastroenterology}

Case Rep Gastroenterol 2020;14:554-560

DOI: $10.1159 / 000511252$

c) 2020 The Author(s). Published by S. Karger AG, Basel www.karger.com/crg

Paparoupa et al.: Checkpoint Inhibitor-Induced Colitis

After a total of 17 infliximab administrations, in overall 12 months, clinical remission of gastrointestinal symptoms, undetectable calprotectin and complete mucosal healing in colonoscopy (Fig. 3) were achieved. Infliximab was therefore discontinued. Administration of mesalazine orally (Salofalk ${ }^{\circledR}$ gastro-resistant prolonged-release granules $3 \mathrm{~g}$ once daily in the morning) is continued until today, since an attempt to discontinuation led to an increase of stool frequency. Hydrocortisone locally (Colifoam ${ }^{\circledR}$ rectal foam) was first tapered to a single application every other day and then applied only as needed which so far was never necessary. A recent colonoscopy showed healthy colonic tissue. The patient is still in full remission of metastatic melanoma, 2 years after discontinuation of nivolumab and ipilimumab.

\section{Discussion}

Dual checkpoint inhibition induced by combination therapy with nivolumab and ipilimumab improves response rates in patients with metastatic melanoma compared to monotherapy. However, a higher rate of toxicity, including immune-related colitis, seems to be present under this regimen showing itself as ICI-induced diarrhea [5, 10]. Diagnostic colonoscopy and multiple colonic biopsies are necessary for the diagnosis of ICI-induced colitis, as a diversity of clinical, endoscopic and histological manifestations have been observed in patients with ICI-induced diarrhea [10]. In our case, erythema, erosions and ulcerations mimicking IBD were described during colonoscopy and histological examination revealed acute inflammatory infiltration of the mucosa with cryptitis and apoptosis. The most prominent macroscopic damage was located in the left colonic segments (sigmoid and rectum) as already described in the literature [11].

High-dose steroid therapy is the milestone in the treatment of ICI-induced colitis, but cases of steroid-refractory colitis have been registered before. Recently, Tidwell and Gutnik [7] have reported on a 66-year-old female with advanced melanoma, who developed a steroid-resistant colitis secondary to administration of nivolumab and ipilimumab, which was successfully treated with infliximab [7]. Our case has many similarities to that of Tidwell and Gutnik, as every time an attempt was made to reduce the daily prednisolone dosage below 30 mg, symptomatic colitis re-emerged.

According to other case reports, protocols of a broad immunosuppressive therapy in patients with refractory colitis under steroids and infliximab have been implemented before. Randhawa et al. [12] have recently presented the case of a 27-year-old female with severe colitis, refractory to methylprednisolone, infliximab and mycophenolate mofetil, who responded to vedolizumab, which is a humanized monoclonal antibody against $\alpha 4 \beta 7$ integrin [12]. On the other hand, Alcantar et al. [13] presented the case of a patient in whom clinical remission was observed under steroids and mycophenolate after high-dose steroids, infliximab, and vedolizumab failed to achieve a resolution of the patients' symptoms [13]. Nevertheless, we decided to keep our immunosuppression regime limited to steroids and infliximab because of the induction of a remarkable clinical remission, even though a daily mesalazine dose is necessary in order to preserve this effect.

The use of local steroids and mesalazine has already been described in the literature as treatment strategies beyond systemic steroids in order to reduce the high burden of side effects. Beclomethasone has already been used in ICI-induced colitis with isolated histological features in the absence of macroscopic disease. Sustained clinical and histological remission was induced without adverse effects [14]. Mesalazine has been implemented in the treatment of relapsed nivolumab-associated colitis as maintenance therapy, as well as remission 


\section{Case Reports in Gastroenterology}

\begin{tabular}{l|l}
\hline Case Rep Gastroenterol 2020;14:554-560 \\
\hline DOI: 10.1159/000511252 & $\begin{array}{l}\text { @ 2020 The Author(s). Published by S. Karger AG, Basel } \\
\text { www.karger.com/crg }\end{array}$ \\
\hline
\end{tabular}

Paparoupa et al.: Checkpoint Inhibitor-Induced Colitis

induction for long-term survival [15]. In our case, mesalazine was well tolerated and successfully administered up to now, in order to maintain remission for a period of more than 2 years.

\section{Conclusion}

Our case shows that ICI-induced colitis can become clinically apparent, even after ICI therapy, in this case with nivolumab and ipilimumab, has been discontinued. Furthermore, we support the notion that infliximab, in combination with mesalazine, can successfully induce a long-lasting remission in patients who have responded to steroids, but clinical deterioration re-emerges by every attempt to achieve a dosage reduction. It is important to note that successful treatment of ICI-induced colitis did not lead to reoccurrence of malignant melanoma after 2 years of follow-up.

\section{Statement of Ethics}

The research was conducted ethically in accordance with the World Medical Association Declaration of Helsinki. A written informed consent for publication, including images, has been obtained from the patient.

\section{Conflict of Interest Statement}

The authors have disclosed that they have no significant relationship with or financial interest in any commercial companies pertaining to this article.

\section{Funding Sources}

No funding was received.

\section{Author Contributions}

Maria Paparoupa and Sophie Stupperich collected all needed data and wrote the manuscript. Lisa Goerg-Reifenberg, Andreas Wittig and Frank Schuppert treated the patient throughout time. Frank Schuppert reviewed the manuscript and provided consultation regarding intellectual argumentation. All authors have read and approved the submitted version of the manuscript.

\section{References}

1 Mearns ES, Bell JA, Galaznik A, Puglielli SM, Cichewicz AB, Boulanger T, et al. Gastrointestinal adverse events with combination of checkpoint inhibitors in advanced melanoma: a systematic review. Melanoma Manag. 2018 Jan;5(1):MMT01.

2 Almutairi AR, McBride A, Slack M, Erstad BL, Abraham I. Potential Immune-Related Adverse Events Associated With Monotherapy and Combination Therapy of Ipilimumab, Nivolumab, and Pembrolizumab for Advanced Melanoma: A Systematic Review and Meta-Analysis. Front Oncol. 2020 Feb;10:91. 


\section{Case Reports in Gastroenterology}

\begin{tabular}{l|l}
\hline DOI: $10.1159 / 000511252$ & $\begin{array}{l}\text { (c) } 2020 \text { The Author(s). Published by S. Karger AG, Basel } \\
\text { www.karger.com/crg }\end{array}$ \\
\hline
\end{tabular}

Paparoupa et al.: Checkpoint Inhibitor-Induced Colitis

3 Hartmann A, Paparoupa M, Volkmer BG, Rompel R, Wittig A, Schuppert F. Autoimmune hypophysitis secondary to therapy with immune checkpoint inhibitors: four cases describing the clinical heterogeneity of central endocrine dysfunction. J Oncol Pharm Pract. 2020 Oct;26(7):1774-79. doi:

$10.1177 / 1078155220910202$.

4 Yoshino K, Nakayama T, Ito A, Sato E, Kitano S. Severe colitis after PD-1 blockade with nivolumab in advanced melanoma patients: potential role of Th1-dominant immune response in immune-related adverse events: two case reports. BMC Cancer. 2019 Oct;19(1):1019.

5 Tian Y, Zhang Z, Yang X, Li D, Zhang L, Li Z, et al. The Risk Ratio of Immune-Related Colitis, Hepatitis, and Pancreatitis in Patients With Solid Tumors Caused by PD-1/PD-L1 Inhibitors: A Systematic Review and Meta-Analysis. Front Oncol. 2020 Feb;10:261.

6 Puzanov I, Diab A, Abdallah K, Bingham CO 3rd, Brogdon C, Dadu R, et al.; Society for Immunotherapy of Cancer Toxicity Management Working Group. Managing toxicities associated with immune checkpoint inhibitors: consensus recommendations from the Society for Immunotherapy of Cancer (SITC) Toxicity Management Working Group. J Immunother Cancer. 2017 Nov;5(1):95.

7 Tidwell C, Gutnik S. Treatment of Immune Checkpoint Inhibitor Induced Colitis with Infliximab. S D Med. 2019 Oct;72(10):454-8.

8 Abu-Sbeih H, Ali FS, Alsaadi D, Jennings J, Luo W, Gong Z, et al. Outcomes of vedolizumab therapy in patients with immune checkpoint inhibitor-induced colitis: a multi-center study. J Immunother Cancer. 2018 Dec;6(1):142.

9 González-Rodríguez E, Rodríguez-Abreu D; Spanish Group for Cancer Immuno-Biotherapy (GETICA). Immune Checkpoint Inhibitors: Review and Management of Endocrine Adverse Events. Oncologist. 2016 Jul;21(7):804-16.

10 Yanai S, Nakamura S, Kawasaki K, Toya Y, Akasaka R, Oizumi T, et al. Immune checkpoint inhibitor-induced diarrhea: clinicopathological study of 11 patients. Dig Endosc. 2020 May;32(4):616-20. https://doi.org/10.1111/den.13555.

11 Wright AP, Piper MS, Bishu S, Stidham RW. Systematic review and case series: flexible sigmoidoscopy identifies most cases of checkpoint inhibitor-induced colitis. Aliment Pharmacol Ther. 2019 Jun;49(12):1474-83.

12 Randhawa M, Gaughran G, Archer C, Pavli P, Morey A, Ali S, et al. Vedolizumab in combined immune checkpoint therapy-induced infliximab-refractory colitis in a patient with metastatic melanoma: A case report. World J Clin Oncol. 2019 Oct;10(10):350-7.

13 Alcantar D, Al-Jaashaami L, Giron F. A Case of Immune Checkpoint Inhibitor Refractory Colitis Treated with Mycophenolate and High-dose Steroids. Cureus. 2019 Dec;11(12):e6392.

14 Ibraheim H, Green M, Papa S, Powell N. Topical beclometasone dipropionate in the management of immune checkpoint inhibitor-induced microscopic colitis. BMJ Case Rep. 2019 Apr;12(4):e226481.

15 Kikuchi H, Sakuraba H, Akemoto Y, Murai Y, Fukutoku Y, Asari T, et al. A case of nivolumab-associated colitis, which relapsed after mucosal healing and was then successfully treated with mesalazine. Immunol Med. 2019 Mar;42(1):39-44.

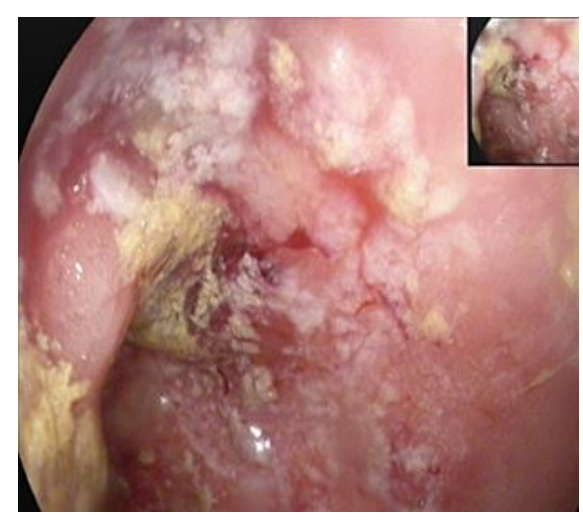

Fig. 1. Diagnostic colonoscopy revealing multiple ulcerative lesions and hemorrhagic colitis of the sigmoid and rectum. 
Case Reports in Gastroenterology

\begin{tabular}{l|l}
\hline Case Rep Gastroenterol 2020;14:554-560 \\
\hline DOI: 10.1159/000511252 & $\begin{array}{l}\text { @ 2020 The Author(s). Published by S. Karger AG, Basel } \\
\text { www.karger.com/crg }\end{array}$ \\
\hline
\end{tabular}

Paparoupa et al.: Checkpoint Inhibitor-Induced Colitis

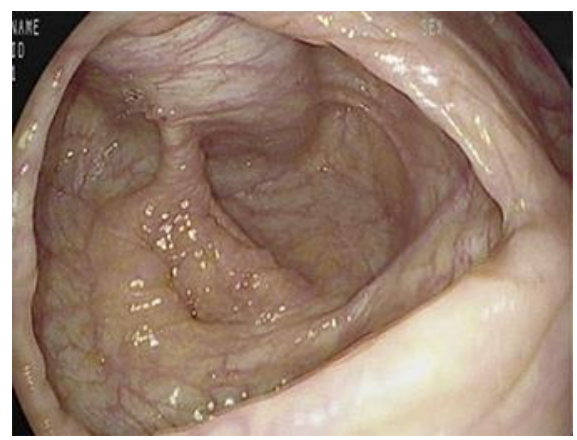

Fig. 2. Terminal ileum and ileocecal valve are macroscopically intact.

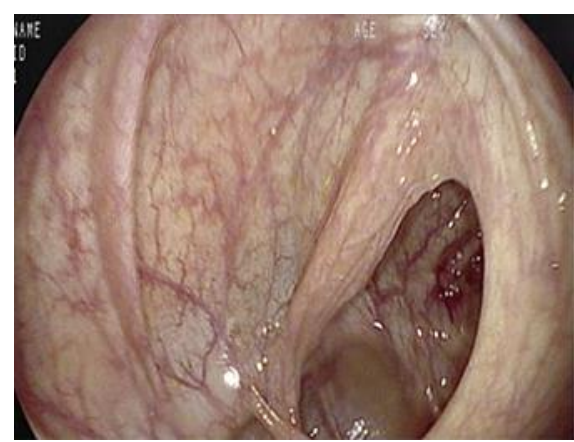

Fig. 3. Diagnostic colonoscopy showing full remission of endoscopic findings under immunosuppressive therapy. 